\title{
Role of Cytogenetic Evaluation in Diagnosis of Acute Myeloid Leukemia
}

\author{
Gadhia Pankaj K. , Patel Monika V., Vaniawala Salil N. \\ Cytogenetic Unit, S. N. Gene Laboratory and Research Centre, Surat, India
}

\section{Email address:}

pankajkgadhia@gmail.com (Gadhia P. K.)

${ }^{*}$ Corresponding author

\section{To cite this article:}

Gadhia Pankaj K., Patel Monika V., Vaniawala Salil N. Role of Cytogenetic Evaluation in Diagnosis of Acute Myeloid Leukemia. American Journal of Biomedical and Life Sciences. Vol. 4, No. 6, 2016, pp. 98-102. doi: 10.11648/j.ajbls.20160406.13

Received: September 21, 2016; Accepted: November 11, 2016; Published: December 14, 2016

\begin{abstract}
Aim: Acute leukaemia represents clonal haematological disorders that arise from at least two or more genetics alteration in susceptible haematological cells. The cytogenetic study confirms a wide variety of common, rare and novel chromosomal anomalies in patients with haematological disorders providing valuable diagnostics and prognostic information. Method: Cytogenetic analyses were carried out in a total 4600 suspected patients. Of which, 68 patients were reported with Acute Myeloid Leukaemia. Cytogenetic analyses from bone marrow cultures having age ranging from 5 years to 65 years were carried out. GTG banded metaphases were analysed and karyotypes by automatic karyotyping system and confirmation were made by using Florescent In Situ Hybridization technique (FISH). Results: Results revealed that out of 68 AML patients only 36 patients $(52.9 \%)$ were found with translocation $\mathrm{t}(8 ; 21)(\mathrm{q} 22 ; \mathrm{q} 22)$ in AML-M2 subtype, 23 patients $(33.8 \%)$ were found with a translocation $\mathrm{t}(15 ; 17)(\mathrm{q} 22 ; \mathrm{q} 12)$ in AML-M3 and only 09 patients $(13.2 \%)$ were found with inversion in chromosome 16 $\operatorname{inv}(16)(\mathrm{p} 13 ; \mathrm{q} 22)$ in AML-M4. Conclusion: It is concluded from the present study that a high prevalence rate of AML were found in $\mathrm{t}(8 ; 21)$ (q22; q22) followed by $\mathrm{t}(15 ; 17)$ (q22; q12) and inv(16) (p13; q22). The significance of results is discussed.
\end{abstract}

Keywords: G-banding, Karyotype, AML, FISH

\section{Introduction}

The nature of so-called complex karyotypic rearrangements associated with malignant hemopathies often undetected on conventional cytogenetics. The FISH technique is a new tool to discover cryptic translocations and to assign the chromosome origin of complex markers [1]". Genetics play an increasingly important role in the risk stratification and management of acute myeloid leukemia (AML) patients. Traditionally, AML classification and risk stratification relied on cytogenetic studies; however, molecular detection of gene mutations is playing an increasingly important role in classification, risk stratification, and management of AML [2]". Acute Myeloid Leukemia (AML) is a heterogeneous group of malignant haematopoietic disorder of rapidly proliferating neoplasm of immature haematopoietic stem cells. Recurrent chromosomal rearrangements such as $\mathrm{t}(8 ; 21)(\mathrm{q} 22 ; \mathrm{q} 22), \mathrm{t}(15 ; 17)$ ( $\mathrm{q} 22$; $\mathrm{q} 12)$, and $\operatorname{inv}(16)(\mathrm{p} 13 \mathrm{q} 22)$ are frequently identified as abnormality in AML. Several studies indicate that the AML patients with normal karyotypes represent the cytogenetically heterogeneous group which correlates with prognosis [3]". AML therapy is not targeted but the intensity of therapy is driven by the prognostic subgroup. Many prognostic scoring systems classify patients into favourable, poor, or intermediate prognostic subgroups based on clinical and genetic features. Current standard of care combines cytogenetic results with targeted testing for mutations in FLT3, NPM1, CEBPA, and KIT to determine the prognostic subgroup. Other gene mutations have also been demonstrated to predict prognosis and may play a role in future risk stratification, although some of these have not been confirmed in multiple studies or established as standard of care [4]". However, World Health Organization in 2008 revised its classification to recognize the impact of molecular markers on prognosis with normal cytogenetic findings, as the most of the patients achieve the complete remission with induction of chemotherapy. Thus, the complete diagnostic and prognostic testing of bone marrow is important to predict 
the outcomes and post induction treatments [5]". Thus, the cytogenetic study is important to confirm the wide variety of common, rare and novel chromosomal anomalies in patients with haematopoietic disorders may provide valuable diagnostic and prognostic information [6]".

Ahmad et al [7]" showed that the t $(8 ; 21)(\mathrm{q} 22 ; \mathrm{q} 22)$ is most common recurrent chromosomal translocation seen in nearly $10-15 \%$ of AML-M2 subtype. While t(15; 17) (q22; q12) found in only $5 \%$ of the specific type of AML such as Acute Promyelocytic Leukaemia(APL) and inv(16) (p13q22) found in approximately $8 \%$ of AML

Velloso et al [6]" have reported that the $50-60 \%$ of patients shown intermediate risk group with chromosomal constitution as $\mathrm{t}(9 ; 11),+8,-\mathrm{Y}$ with normal karyotype, while $25-30 \%$ patients shown poor risk group with MLL, $t(6 ; 9)$, monosomy and deletion of chromosome 5 and 3 , inv(3) and $25 \%$ of patients belong to good risk group with cytogenetic chromosomal constitution as $\mathrm{t}(15 ; 17)(\mathrm{q} 22 ; \mathrm{q} 12), \mathrm{t}(8 ; 21)$ (q22; q22) and inv (16) (p13; q22).

In the present study, we describe the role of cytogenetic evaluation in the diagnosis of AML, as it is an important prognostic marker in the management of AML along with recognition of specific subtypes. We also report, the prevalence rate of cytogenetic abnormality found in adults and its incidence increases with age.

\section{Materials and Methods}

A total of 4600 Patients were screened from January 2015 to December 2015 who were suspected to have AML. Conventional Cytogenetic was performed on 24 hours unstimulated short term culture of bone marrow cells. The cells were grown in culture medium Marrow Max (GIBCO) supplemented with $20 \%$ of Fetal Bovine Serum (FBS). The colcemid was added for 30 minutes followed by KCL (75 $\mathrm{mM}$ ) at room temperature for $27 \mathrm{~min}$ and Carnoy's fixative for four times. Slides were stained with Giemsa Trypsin Giemsa (GTG) banding technique. GTG banded metaphases from each culture were analysed and karyotyped by using automatic IKAROS karyotyping software. The karyotypes were described according to International system for human cytogenetic nomenclature [8.]" The cytogenetic findings were confirmed by using FISH.

\section{Results}

In the present investigation total of 4600 AML suspected patients were studied. Of which, 68 were reported with AML. Among 68 patients, 36(52.9\%) were reported with $t(8$; 21) (q22; q22) in AML-M2 subtype, 23(33.8\%) were with a $\mathrm{t}(15 ; 17) \quad(\mathrm{q} 22 ; \mathrm{q} 12)$ in AML-M3 and only 09 patients $(13.2 \%)$ were found with inversion in chromosome16 inv(16) (p13; q22) in AML-M4. The abnormal karyotypes with complex translocations are shown in [Figures 1, 2, and $3]$ ". The results suggest that a high prevalence rate of AML were found in $\mathrm{t}(8 ; 21)$ (q22; q22) followed by $\mathrm{t}(15 ; 17)$ (q22; $\mathrm{q} 12)$ and $\operatorname{inv}(16)(\mathrm{p} 13 ; \mathrm{q} 22)$ respectively [Figure 4]".
The results of dual colour FISH analysis using AML1 and ETO probes are shown in Figures 5 and 6" In Figure 5 there are two separate red and green signals showing normal chromosomes, while Figure 6 clearly shows two fusions, one red and one green signals of AML1/ETO confirming $\mathrm{t}(8 ; 21)$. While using PML and RARA probes, Figure 7 showed two separate signals red for PML and green for RARA. While Figure 8 , showed two fusions with red, green and yellow indicating $\mathrm{t}(15 ; 17)$. Interstitial, FISH analysis using inv(16) shown in Figure 9 with two fusions of red and green signals indicating normal chromosome \#16. While Figure 10 shows fusion of red and green signals in one which is normal chromosome \#16 and separate green and red signals indicating inv(16) chromosome.

\section{Discussion}

Acute myeloid leukemia (AML) represents a group of clonal hematopoietic stem cells disorders that is characterized by both failure to differentiate and over proliferation of the stem cell compartment by non-functional cells called myelobalasts at the expense of normal cells [9.]" Cytogenetic analysis plays a critical role in the diagnosis, classification, prognosis, and management of acute myeloid leukemia (AML). It has become an essential technique that helps doctors identify leukemia and provide treatment guidance.

Chromosomal abnormalities in AML classified as numerical and structural, chromosomal abnormality can be defined as disturbances in normal composition of chromosomes. Numerical abnormalities take the form of an aberrant copy number of particular chromosomes. This phenomenon occurs due to the fact that chromosome missegregation takes place when the cell divides resulting in the loss or gain of specific chromosomes [10.]"

If AML is untreated, most patients will die over a period of days or weeks based largely on the level of blasts in the blood and bone-marrow. Cytogenetics is recognized as one of the most important valuable prognostic determinators in AML. An abnormal karyotype has been found in approximately $60 \%$ of AML patients have favourable cytogenetics that involve $\mathrm{t}(8 ; 21), \mathrm{t}(15 ; 17)$ and inv(16); these patients have complete remission (CR) rate over $90 \%$ and five year survival of $65 \%$ [11]"

The aim of the study was to evaluate a role of cytogenetic in the diagnosis of AML A large numbers of AML suspected patients were screened. Overall AML prevalence rates of $15 \%$ were observed in AML M2, M3 and M4e0 subtypes which are similar to previously reported study. But, in the present study $\mathrm{t}(8 ; 21)(52.9 \%), \mathrm{t}(15 ; 17)(33.82 \%)$ and $\operatorname{inv}(16)(13.23 \%)$ were higher as compared to those reported by the $10 \%, 10 \%$ and $5 \%$ in $\mathrm{t}(8 ; 21), \mathrm{t}(15 ; 17)$ and $\operatorname{inv}(16)$ respectively [12]". 


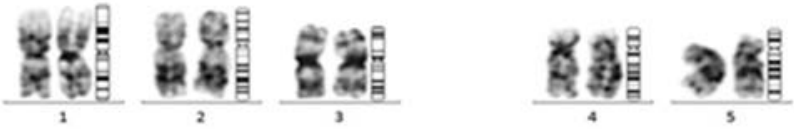 \\ 3)

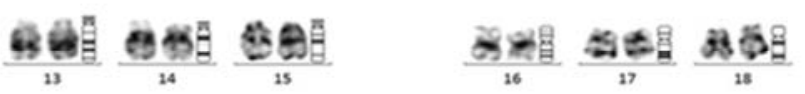

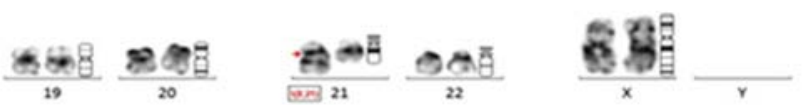

Figure 1. G-banded karyotype of a female AML patient $t(8 ; 21)(q 22 ; q 22)$.

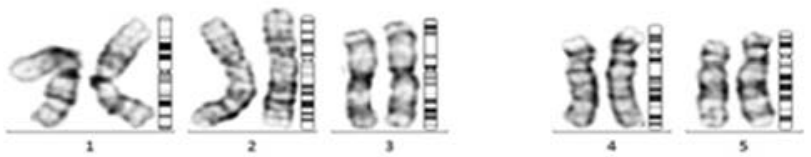

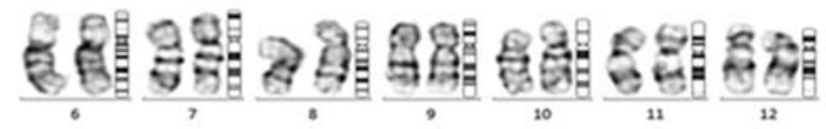

E⿱㇒日勺:

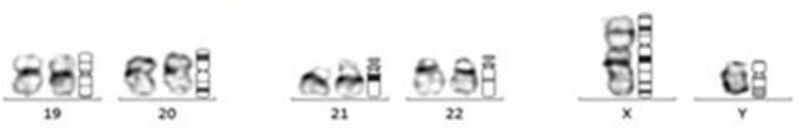

Figure 2. G-Banded karyotype of a male patient showing $t(15 ; 17)$ (q22; q12.).

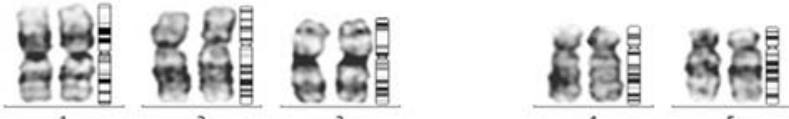

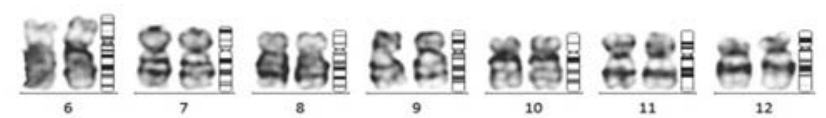

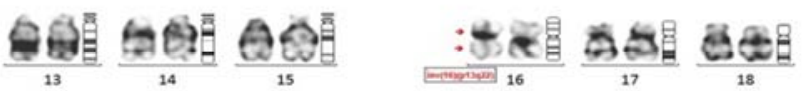

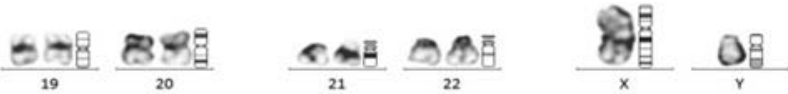

Figure 3. G-Banded Karyotype of Male AML Patient Showing inv(16) (p13; q22).

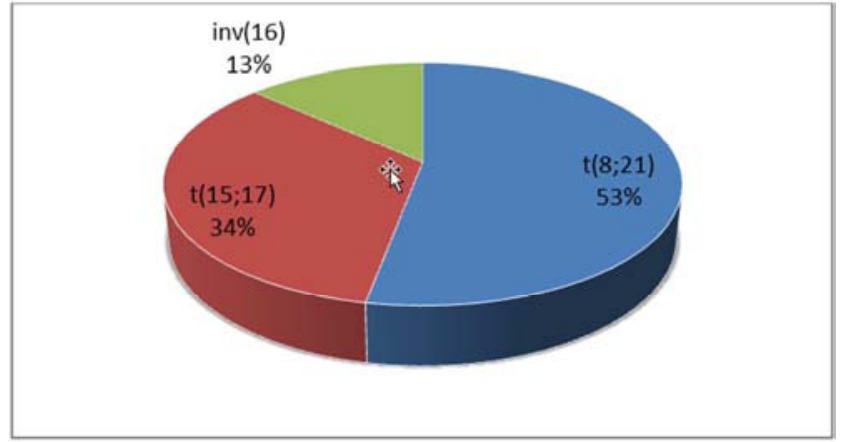

Figure 4. Frequncy of Prevelance rate found in $A M L$.

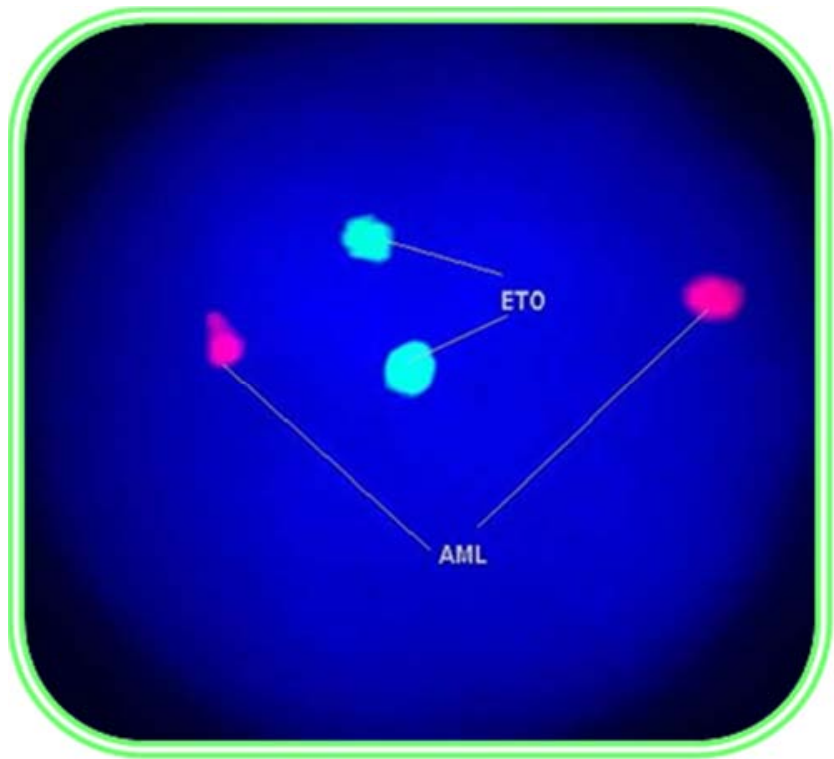

Figure 5. FISH showing AML(red)and ETO (green).

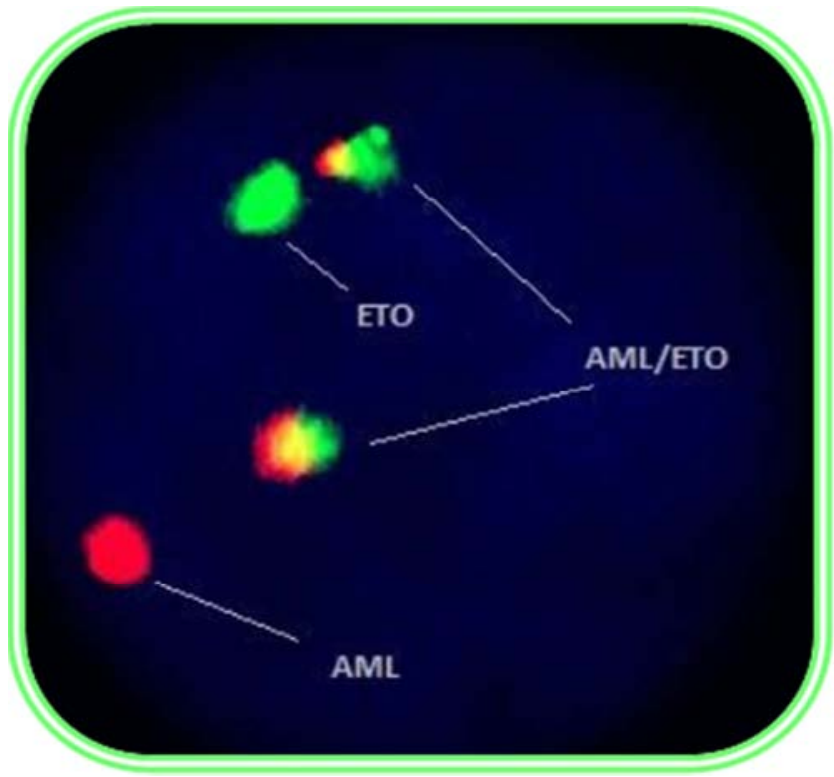

Figure 6. FISH analysis showing AML1/ETO (red and green) fusion signals. 


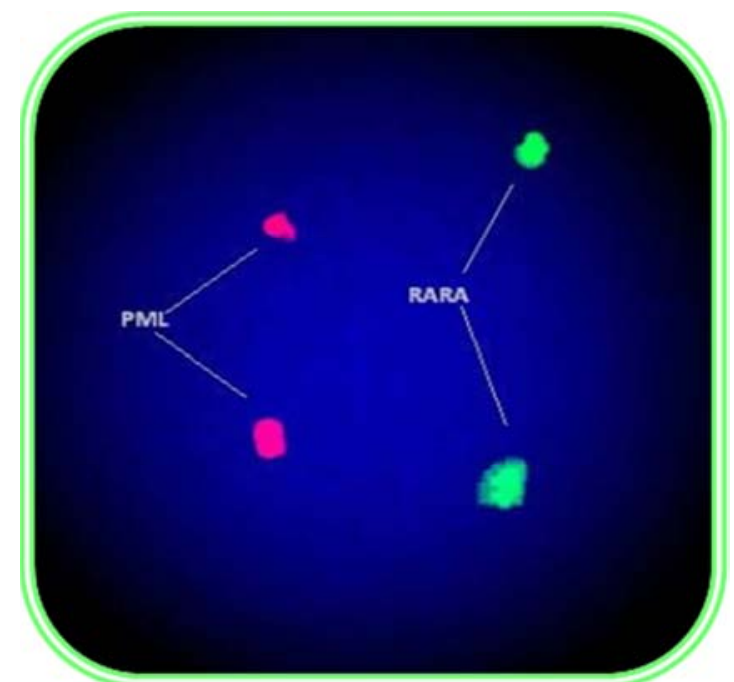

Figure 7. FISH analysis showing PML (red) and RARA (green).

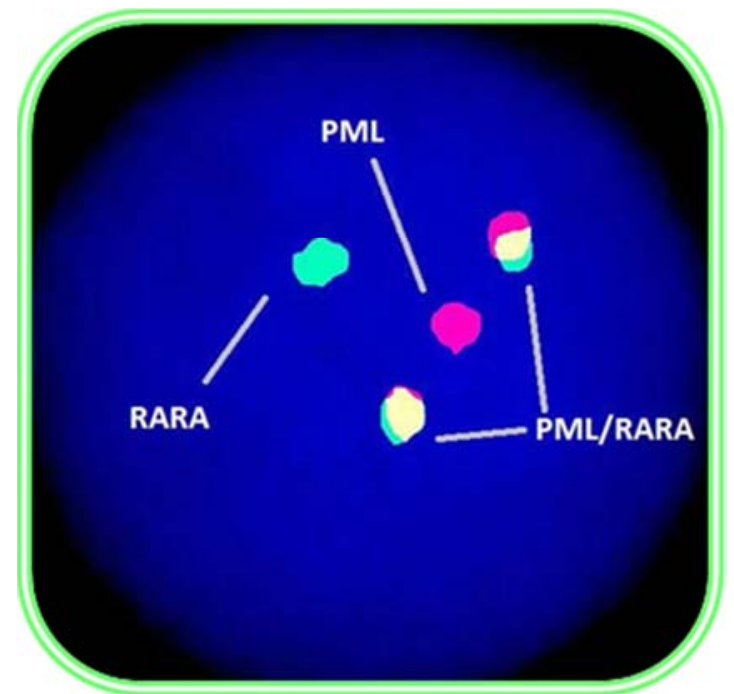

Figure 8. FISH analysis showing PML/RARA (green, red and yellow fusion signal.

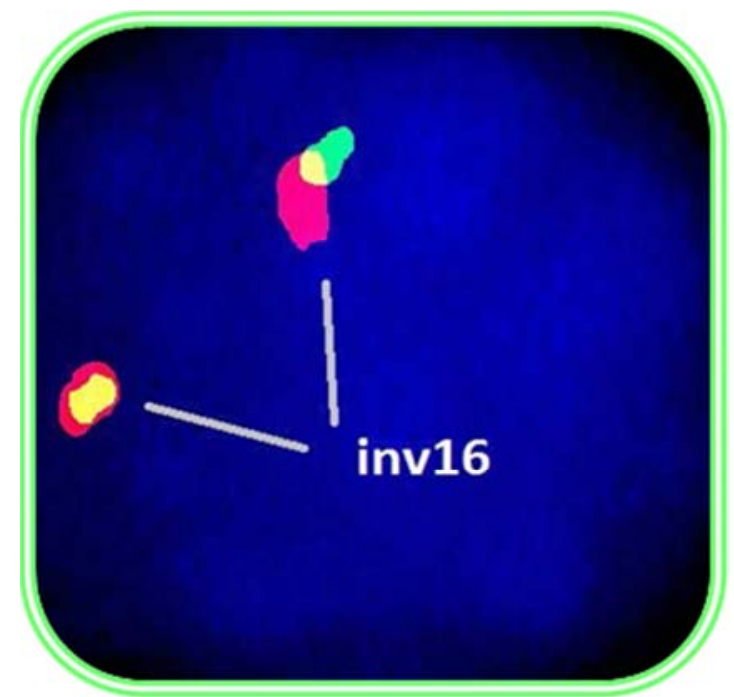

Figure 9. FISH analysis showing fusion of green and red in normal 16 chromosome.

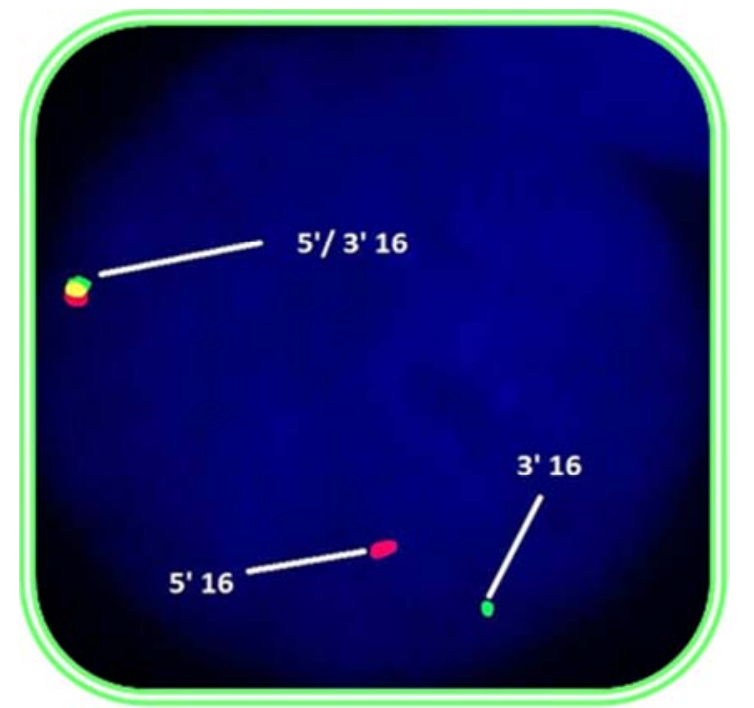

Figure 10. FISH analysis showing one green and red fusion in in normal 16 and separate red and green signals indicate inv(16) chromosome.

Identification of $\mathrm{t}(8 ; 21)(\mathrm{q} 22 ; \mathrm{q} 22), \mathrm{t}(15 ; 17)(\mathrm{q} 22 ; \mathrm{q} 12)$, and inv(16) (p13; q22) or its molecular equivalent rearrangement of genes is considered to be most valuable tool for cytogenetic and FISH studies [13]". Thus, for diagnosis, a FISH was more sensitive and accurate than conventional cytogenetic in detecting rearrangements besides confirming abnormality. Thus, all AMLs type namely AML $\mathrm{M} 2, \mathrm{M} 3$ and $\mathrm{M} 4 \mathrm{e} 0$ are considered to be a good risk factor for prognosis.

\section{Conclusion}

Cytogenetics is considered one of the most valuable prognostic determinants in AML. In the present study, a total of 4600 patients were analysed with different age-groups. AML associated with $\mathrm{t}(8 ; 21), \mathrm{t}(15 ; 17)$ and inv(16) predicted as a good-risk group in comparison to the complex karyotypes. For good-risk group, an autologous or allogeneic SCT should be reserved for patients who relapsed after chemotherapy. The study highlights the importance of diagnostic cytogenetics as an independent prognostic factor in AML.

\section{References}

[1] SL. Starza, C. Matteucci, B. Crescenzi, G. Perla, M. Carotenuto, MF. Martelli et al., "Identification of chromosome changes in Acute myeloid leukemia (AML-L2) by Molecular cytogenetics" Can Genet Cytogenet, 1997, 95, pp. 148-152.

[2] S Yohe, "Molecular Genetic markers in Acute leukemia". J. Clin. Med., 2015, 4: pp. 460-478.

[3] S. Frohling, RF. Schlenk, J. Breitruck, A. Benner, KS. Tobis, H. Dohner and K. Dohner, "Prognostic significance of activating FLT3 mutations in younger adults (16 to 60 years) with acute myeloid leukemia and normal cytogenetics: a study of AML study Group Ulm", Blood, 2002, 100: pp. 4372-4380. 
[4] O. Blau, R. Berenstein, A. Sindram, IW. Blau. "Molecular analysis of different FLT3-ITD mutations in acute myeloid leukemia". Leuk. Lymphoma 2013, 54, pp. 145-152.

[5] TL. Lin, T. Williams, J. He, OS. Aljitawi, S. Ganguly, S. Abhyankar, S. Fleming, H. Male and JP. McGuirk, "Rates of complete diagnostic testing for patients with acute myeloid leukemia" Cancer Mdicine, 2015, 4(4): pp. 519-522.

[6] ED. Velloso, CH, Motta, JB. Furtado, NS. Bacal, PA. Silveira, CB. Moyses, R. Sitnik, and JR. Pinho, "Molecular and cytogenetic abnormalities in acute myeloid leukemia: Review and case studies", Einstein, 2011, 9(2), pp. 184-189.

[7] F. Ahmed, P. Kokte, P. Chedda, R. Dalvi, B. Ranjandas and S. Mandava, "Molecular cytogenetic findings in a three-way novel variant of $\mathrm{t}(1 ; 8 ; 21)(\mathrm{p} 35 ; \mathrm{q} 22 ; \mathrm{q} 22)$ a unique relocation of the AML1/ETO fusion gene 1p35 in AML-M2", Cancer Genet. Cytogenet., 2008, 180: pp. 153-157.

[8] ISCN, International System for Human Cytogenetics Nomenclature (ISCN), 2009, S. Karger Publ. Inc.

[9] RM, Stone, MR, O’Donnell, MA, Sekeers, “Acute myeloid leukemia", Am. Soc. Hematol. Edu. Program, 2004, pp. 98117.

[10] B, Williams, A, Amon, (2009). Aneuploidy: Cancer's Fatal Flaw?. Cancer Research, 69(13), pp. 5289-5291.

[11] D, Grimwade, RK, Hills, AV, Moorman, H, Walker S, Chatters, AH, Goldstone, AK, Burnett, "Refinement of cytogenetic classification in acute myeloid leukaemia: determination of prognostic significance of rare recurring chromosomal abnormalities among 5876 younger adult patients treated in the United Kingdom Medical Research Council Trials" Blood, 116(3), pp 354-365.

[12] C. Chandra Kumar, "Genetic abnormality and challenges in the treatment of Acute myeloid leukemia", Gene and Cancer, 2011, 2(2): pp. 95-107.

[13] MLLF. Chauffaile, MS. Figueiredo, R. Beltrani, R. Antunes, SV. Yamomoto and J. Kerbauy, "Acute promyelocytic leukemia: the study of $\mathrm{t}(15 ; 17)$ transloction by fluorescent in situ hybridization, reverse trancriptase polymerase chain reaction and cytogenetic techniques" Brazilian J. Medical and Biological Research, 2001, 34: pp. 735-743. 